\title{
Effects of Fly Ash Microsphere on Flowability and Cohesiveness of Cement Paste
}

\author{
Jia-Jian $\mathrm{CHEN}^{1, \mathrm{a}^{*}}$, Pui-Lam NG ${ }^{2,3, \mathrm{~b}}$, Leo Gu LI ${ }^{4, \mathrm{c}}$, Albert Kwok-Hung KWAN ${ }^{3, \mathrm{~d}}$ \\ ${ }^{1}$ Department of Civil Engineering, Foshan University, Foshan, China \\ ${ }^{2}$ Vilnius Gediminas Technical University, Vilnius, Lithuania \\ ${ }^{3}$ Department of Civil Engineering, The University of Hong Kong, Hong Kong, China \\ ${ }^{4}$ Department of Civil Engineering, Guangdong University of Technology, Guangzhou, China \\ achen.jiajian@yahoo.com.hk, birdngpl@gmail.com, ${ }^{\mathrm{c} l i g u 123 @ m s n . c o m, ~}{ }^{\mathrm{d}}$ khkwan@hku.hk \\ ${ }^{*}$ Corresponding author
}

Keywords: Cement paste, Cohesiveness, Fly ash microsphere, Flowability.

\begin{abstract}
This study evaluates the effects of fly ash microsphere (FAM) on the flowability and cohesiveness performance of cement paste. FAM is a superfine fly ash captured from the coal-fired power stations. It has a mean particle size of around $2 \mu \mathrm{m}$ and is of spherical shape. An experimental program comprising a total of 38 cement paste mixes was launched and the flowability and cohesiveness performance of the mixes were measured. Results showed that addition of FAM up to $40 \%$ by volume could improve the flowability but decrease the cohesiveness at the same water to cementitious materials (W/CM) ratio. In general, the cohesiveness of cement paste would decrease with increasing flowability.
\end{abstract}

\section{Introduction}

The workability and cohesivensss of cement or cementitious paste phase of concrete are closely related to the packing density. Since the water has to first fill up the voids in the bulk volume of cementitious materials and the excess water (water in excess of that needed to fill the voids) forms water films coating the particles to lubricate the cement paste, a higher packing density of the cementitious materials would improve the flowability for a given volume of water, and reduce the volume of water needed for a given flowability requirement [1,2]. The packing density improvement due to addition of fillers depends very much on the fineness of the fillers. For maximum packing density, a continuous particle size distribution is preferred.

As a common practice, fly ash and microsilica are often added to the cement paste to improve various properties of the concrete produced [3]. However, the ternary blending of ordinary Portland cement (OPC), fly ash and microsilica might not achieve continuous particle size distribution. In general, the sizes of OPC and fly ash particles are in the order of $10 \mu \mathrm{m}$, while the size of microsilica is in the order of $0.1 \mu \mathrm{m}$. Hence, there is a gap in between 0.5 and $5 \mu \mathrm{m}$ size range. The size distribution of the cement paste phase could be improved by filling up the gap with filler particles of suitable size.

To fill the gap between 0.5 and $5 \mu \mathrm{m}$, fly ash microsphere (FAM) is a feasible choice. FAM is a superfine fly ash captured from the exhaust smoke of coal-fired power stations [4,5]. It has a mean particle size of around $2 \mu \mathrm{m}$ and is highly spherical in shape. It is finer than OPC and fly ash but is coarser than microsilica. The following benefits are expected from the use of FAM: (1) increase the packing density so as to enhance the workability and durability performances of concrete; (2) make use of the otherwise waste material to mitigate the environmental problems associated with FAM disposal; and (3) reduce the amount of cement needed, and thus reduce the energy consumption and carbon footprint of concrete production. To verify the effects of addition of FAM on flowability and cohesiveness performance, a systematic study has been carried out, as reported herein. 


\section{Materials}

The OPC was of strength grade 52.5N and it complied with British Standard BS 12: 1996, whereas the FAM complied with Chinese Standard GB/T 1596-91. The solid densities of the OPC and FAM were measured to be $3112 \mathrm{~kg} / \mathrm{m}^{3}$ and $2520 \mathrm{~kg} / \mathrm{m}^{3}$, respectively. Their particle size distributions were measured by a laser diffraction particle size analyzer with ultrasonic vibration applied to disperse the particles and the results so obtained are plotted in Fig. 1. From these results, the mean particle sizes of the OPC and FAM were computed as $14.1 \mu \mathrm{m}$ and $2.6 \mu \mathrm{m}$, respectively.

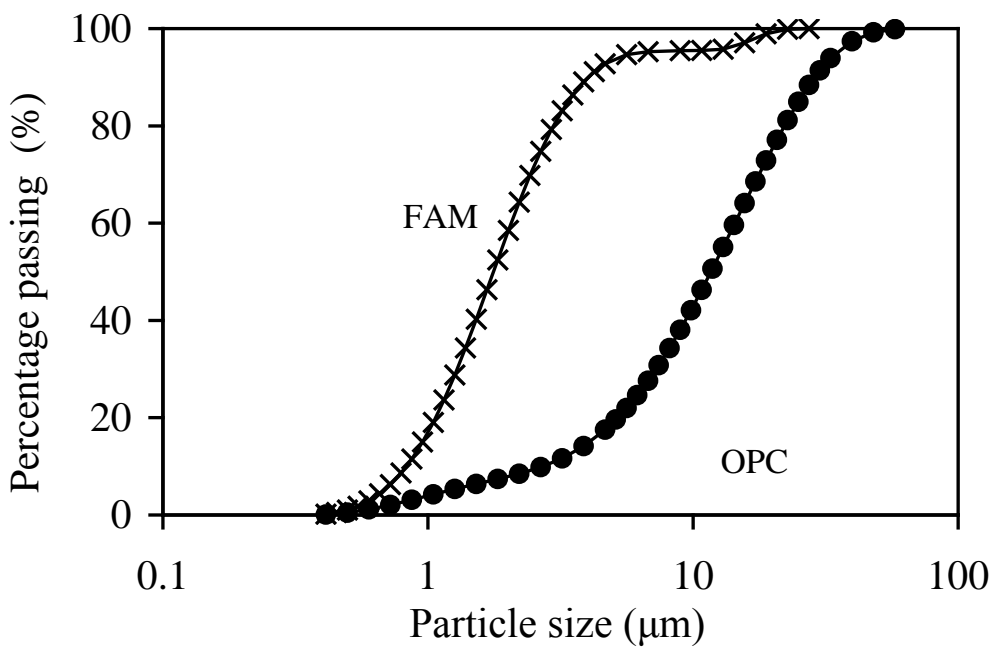

Fig. 1 Particle size distributions of OPC and FAM

\section{Experimental Program}

A total of 38 cement paste mixes containing various amounts of FAM at different water to cementitious materials (W/CM) ratios were produced and their flow spread, flow rate and cohesiveness were measured. The FAM content was varied from $0 \%$ to $60 \%$ by volume in increments of $20 \%$ and the W/CM ratio was varied between 0.10 and 0.30 by mass. Some mixes at low W/CM ratios were too dry to form a workable paste and thus their flowability measurements were not carried out. The mix proportions of the cement paste mixes are summarized in Table 1.

\section{Test Methods}

Measurement of flow spread and flow rate. The mini slump cone test and Marsh cone test were used to measure the flow spread and flow rate, respectively, of the cement paste samples. The mini slump cone adopted was the same as that used by Okamura and Ouchi [6] whereas the Marsh cone adopted was the same as that specified in BS EN 445: 2007 and used in previous studies [1,2]. In conducting the mini slump cone test, the flow spread was determined as the average diameter of the cement paste patty formed minus the base diameter of the slump cone. In conducting the Marsh cone test, the flow rate was determined as the volume of cement paste divided by the flow time out of the Marsh cone.

Measurement of cohesiveness. There is up to now no established test method for measuring the cohesiveness of cement paste. In this study, the viability of using a mini version of the sieve segregation test to measure the cohesiveness of cement paste in terms of sieve segregation indices was explored. Two sieves, one of $0.6 \mathrm{~mm}$ aperture size and the other of $1.18 \mathrm{~mm}$ aperture size, were employed for the sieve segregation tests. These two sieves are same as those normally used for sieve analysis of fine aggregate. Apart from the aperture size, they are similar to each other and both have 
an overall diameter of $200 \mathrm{~mm}$. The percentage of the cement paste that can penetrate the aperture of sieve, called sieve segregation index (SSI), was determined and used as an indication of the cohesiveness of the cement paste. A low SSI represents a high cohesiveness whereas a high SSI represents a low cohesiveness.

Table 1 Mix proportions of cement paste mixes

\begin{tabular}{|c|c|c|c|c|c|c|}
\hline Mix no. & $\begin{array}{c}\text { FAM } \\
\text { percentage } \\
(\%)\end{array}$ & W/CM ratio & $\begin{array}{c}\text { OPC } \\
\text { content } \\
\left(\mathrm{kg} / \mathrm{m}^{3}\right)\end{array}$ & $\begin{array}{c}\text { FAM } \\
\text { content } \\
\left(\mathrm{kg} / \mathrm{m}^{3}\right)\end{array}$ & $\begin{array}{c}\text { Water } \\
\text { content } \\
\left(\mathrm{kg} / \mathrm{m}^{3}\right)\end{array}$ & $\begin{array}{c}\text { SP dosage } \\
\left(\mathrm{kg} / \mathrm{m}^{3}\right)\end{array}$ \\
\hline 1 & 0 & 0.16 & 2071 & 0 & 316 & 19 \\
\hline 2 & 0 & 0.18 & 1988 & 0 & 343 & 18 \\
\hline 3 & 0 & 0.20 & 1912 & 0 & 368 & 18 \\
\hline 4 & 0 & 0.22 & 1842 & 0 & 392 & 17 \\
\hline 5 & 0 & 0.24 & 1776 & 0 & 413 & 16 \\
\hline 6 & 0 & 0.26 & 1716 & 0 & 433 & 16 \\
\hline 7 & 0 & 0.28 & 1659 & 0 & 452 & 15 \\
\hline 8 & 0 & 0.30 & 1605 & 0 & 470 & 15 \\
\hline 9 & 20 & 0.14 & 1750 & 354 & 268 & 30 \\
\hline 10 & 20 & 0.16 & 1679 & 340 & 297 & 29 \\
\hline 11 & 20 & 0.18 & 1614 & 327 & 325 & 28 \\
\hline 12 & 20 & 0.20 & 1553 & 314 & 350 & 27 \\
\hline 13 & 20 & 0.22 & 1497 & 303 & 373 & 26 \\
\hline 14 & 20 & 0.24 & 1445 & 293 & 395 & 25 \\
\hline 15 & 20 & 0.26 & 1397 & 283 & 415 & 24 \\
\hline 16 & 20 & 0.28 & 1351 & 274 & 434 & 24 \\
\hline 17 & 20 & 0.30 & 1309 & 265 & 452 & 23 \\
\hline 18 & 40 & 0.10 & 1447 & 781 & 182 & 45 \\
\hline 19 & 40 & 0.12 & 1385 & 748 & 216 & 43 \\
\hline 20 & 40 & 0.14 & 1329 & 717 & 249 & 41 \\
\hline 21 & 40 & 0.16 & 1276 & 689 & 278 & 40 \\
\hline 22 & 40 & 0.18 & 1228 & 663 & 305 & 38 \\
\hline 23 & 40 & 0.20 & 1183 & 639 & 331 & 37 \\
\hline 24 & 40 & 0.22 & 1142 & 616 & 354 & 35 \\
\hline 25 & 40 & 0.24 & 1103 & 595 & 376 & 34 \\
\hline 26 & 40 & 0.26 & 1067 & 576 & 397 & 33 \\
\hline 27 & 40 & 0.28 & 1033 & 558 & 416 & 32 \\
\hline 28 & 40 & 0.30 & 1001 & 540 & 434 & 31 \\
\hline 29 & 60 & 0.12 & 934 & 1134 & 197 & 54 \\
\hline 30 & 60 & 0.14 & 897 & 1089 & 229 & 52 \\
\hline 31 & 60 & 0.16 & 863 & 1048 & 258 & 50 \\
\hline 32 & 60 & 0.18 & 831 & 1009 & 286 & 48 \\
\hline 33 & 60 & 0.20 & 801 & 973 & 311 & 47 \\
\hline 34 & 60 & 0.22 & 774 & 940 & 335 & 45 \\
\hline 35 & 60 & 0.24 & 748 & 909 & 357 & 44 \\
\hline 36 & 60 & 0.26 & 724 & 880 & 377 & 42 \\
\hline 37 & 60 & 0.28 & 702 & 852 & 397 & 41 \\
\hline 38 & 60 & 0.30 & 681 & 827 & 415 & 40 \\
\hline
\end{tabular}

\section{Test Results}

Flow spread. The effects of FAM on the flow spread is shown in Fig. 2. Comparing the flow spread versus W/CM ratio curves for different FAM contents, it is evident that the addition of FAM up to $40 \%$ always increased the flow spread of the cement paste. Such increase in flow spread was proportionally larger at lower W/CM ratio. For instance, the addition of $20 \%$ and $40 \%$ FAM 
increased the flow spread from zero to 145 and $210 \mathrm{~mm}$, respectively, at a W/CM ratio of 0.16; and increased the flow spread from $347 \mathrm{~mm}$ to 370 and $409 \mathrm{~mm}$, respectively, at a W/CM ratio of 0.30 . However, further addition of FAM to beyond $40 \%$ always decreased the flow spread.

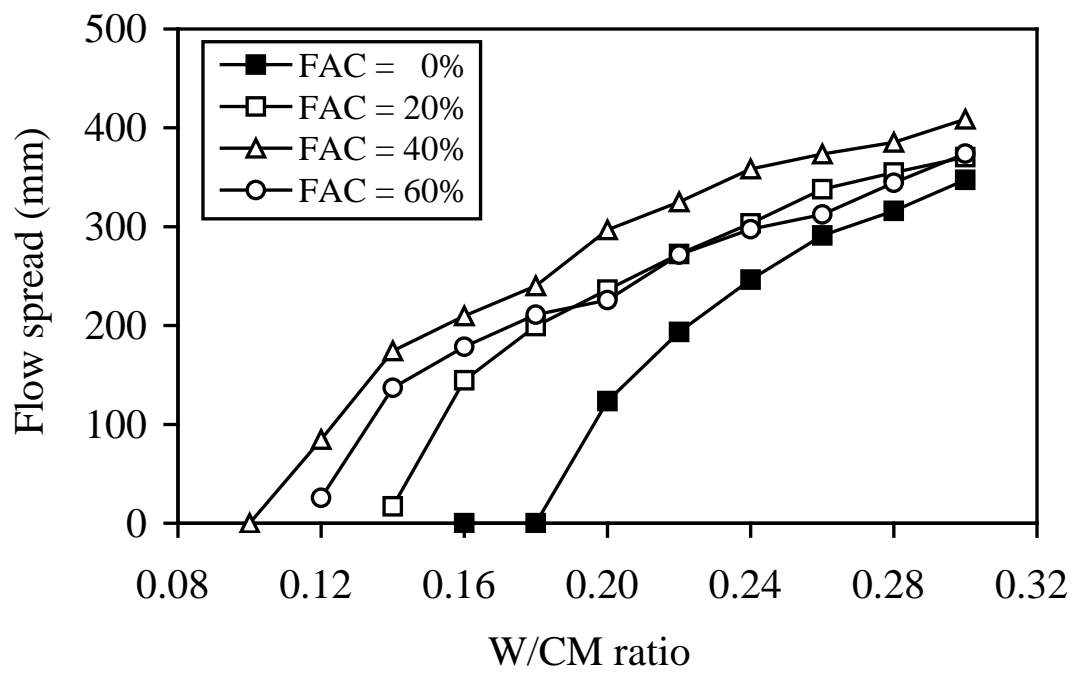

Fig. 2 Effects of FAM on flow spread

Flow rate. The effects of FAM on the flow rate is shown in Fig. 3. Comparing the flow rate versus W/CM ratio curves for different FAM contents, it can be seen that the addition of FAM up to 40\% always increased the flow rate of the cement paste. For instance, the addition of $20 \%$ and $40 \%$ FAM increased the flow rate from zero to 2.2 and $5.7 \mathrm{ml} / \mathrm{s}$, respectively, at a W/CM ratio of 0.16 ; and increased the flow rate from $23.3 \mathrm{ml} / \mathrm{s}$ to 29.3 and $43.4 \mathrm{ml} / \mathrm{s}$, respectively, at a W/CM ratio of 0.30 . However, further addition of FAM to beyond 40\% may slightly decrease the flow rate.

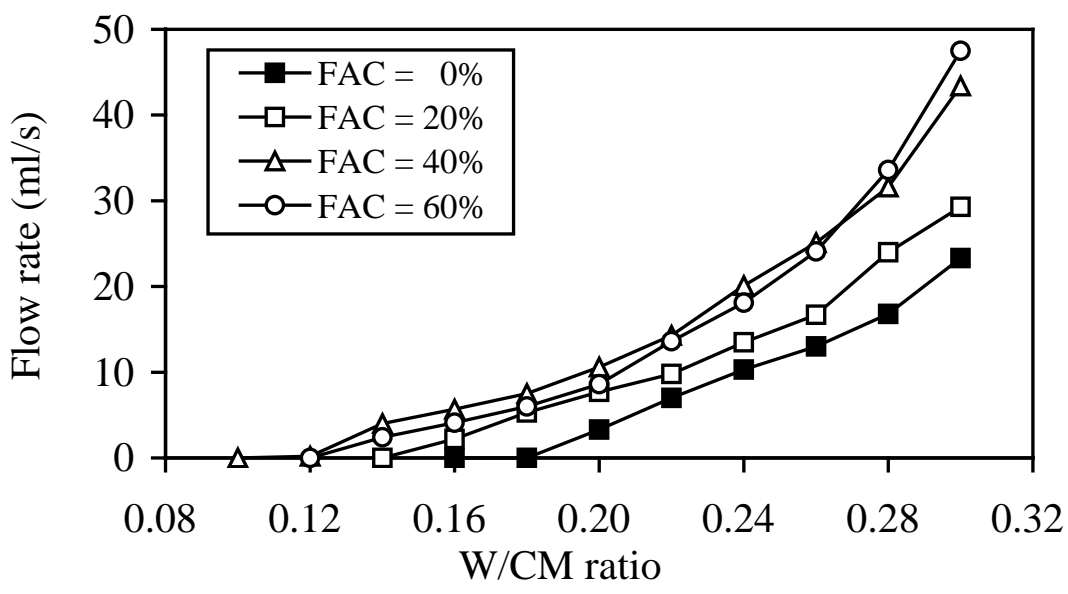

Fig. 3 Effects of FAM on flow rate

The flow rate, a dynamic flowability index, was plotted against the flow spread, a semi-dynamic flowability index, for different FAM contents in Fig. 4. It shows that the dynamic flowability measurememnt results are generally positively correlated with the semi-dynamic flowability measurment results. Such correlation proves that both the mini slump cone test and Marsh cone test are feasible and representative tests for the flowability measurement. It can be seen from Fig. 4 that, at low flowability, the relationship between the dynamic flowability index and the semi-dynamic flowability index is not much dependent on the FAM contents; whereas at a high flowability, the dynamic flowability index increased at a greater rate than the semi-dynamic flowability index for 
$60 \%$ FAM. This may be attributed to the ball bearing effect of the FAM particle, which acts like a ball bearing when the cement paste flows.

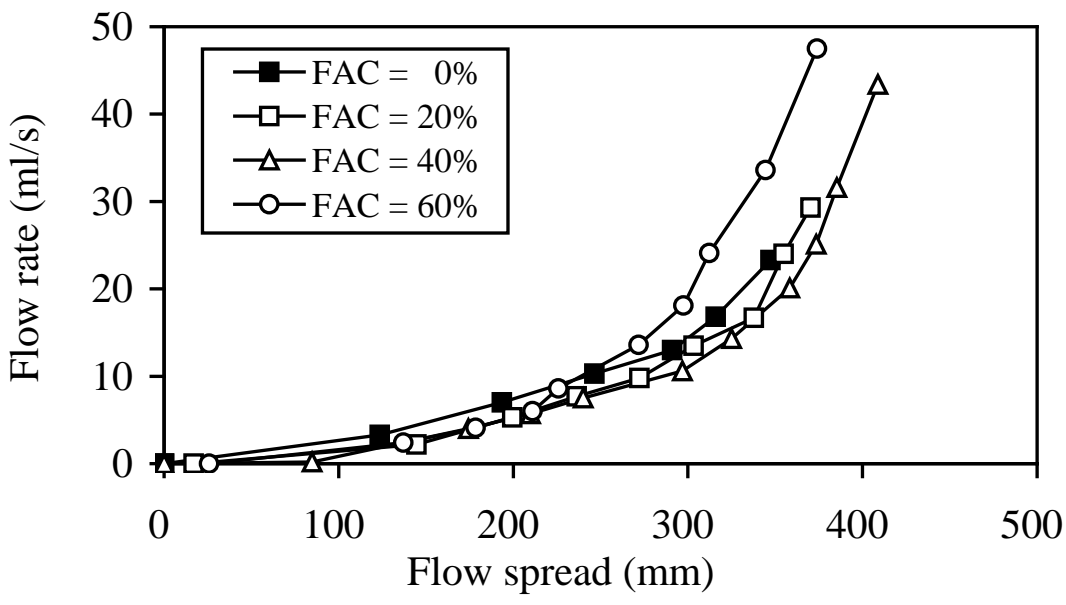

Fig. 4 Relationship between flow spread and flow rate

Cohesiveness. The effects of FAM on the cohesiveness is shown in Fig. 5. Comparing both the 0.6 $\mathrm{mm}$ and $1.18 \mathrm{~mm}$ SSI versus W/CM ratio curves for different FAM contents, it can be seen that the addition of FAM up to $40 \%$ always increased the SSI, in other words, decrease the cohesiveness of the cement paste. For instance, at a W/CM ratio of 0.18 , the addition of $20 \%$ and $40 \%$ FAM increased the $0.6 \mathrm{~mm}$ SSI from zero (when no FAM was added) to $36.0 \%$ and $56.1 \%$, respectively; whereas the addition of $20 \%$ and $40 \%$ FAM increased the $1.18 \mathrm{~mm}$ SSI from zero (when no FAM was added) to $46.5 \%$ and $64.6 \%$, respectively. However, further addition of FAM to beyond $40 \%$ would slightly decrease both the $0.6 \mathrm{~mm}$ and $1.18 \mathrm{~mm}$ SSI, in other words, increase the cohesiveness of the cement paste. It is noteworthy that the numerical values of SSI of cementitious paste are not directly comparable to the SSI of mortar and concrete mixes in assessment of segregate stability. Further research is recommended to establish the relationships between these sieve segregation indices.

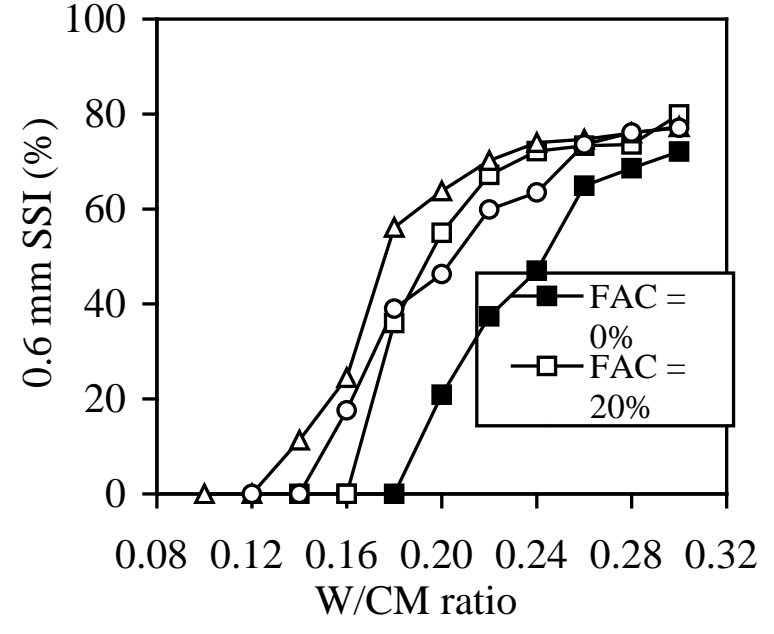

(a) $0.6 \mathrm{~mm} \mathrm{SSI}$

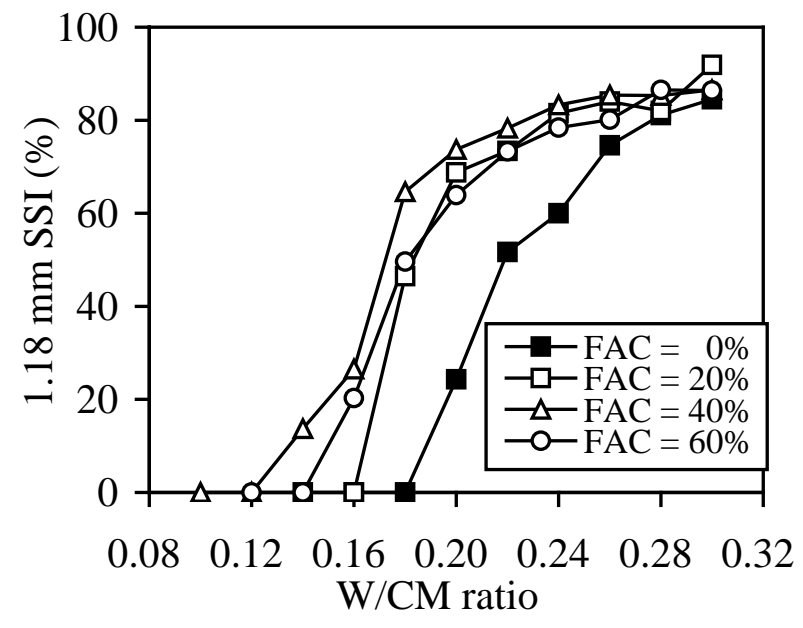

(b) $1.18 \mathrm{~mm}$ SSI

Fig. 5 Effects of FAM on cohesiveness

\section{Concurrent Cohesiveness-Flowability Performance}

The effects of adding FAM on the cohesiveness-flowability performance of cement paste are illustrated by plotting the SSI against the flowability (flow spread or flow rate) in Fig. 6. Each performance curve is a plot of the cohesiveness and flowability that could be concurrently achieved 
by the cement paste at certain FAM content. It can be seen from Fig. 6 that generally the cohesiveness would decrease with the flowability. With the addition of FAM, a higher cohesiveness occurred at a low flowability while a slightly lower cohesiveness occurred at a high flowability.
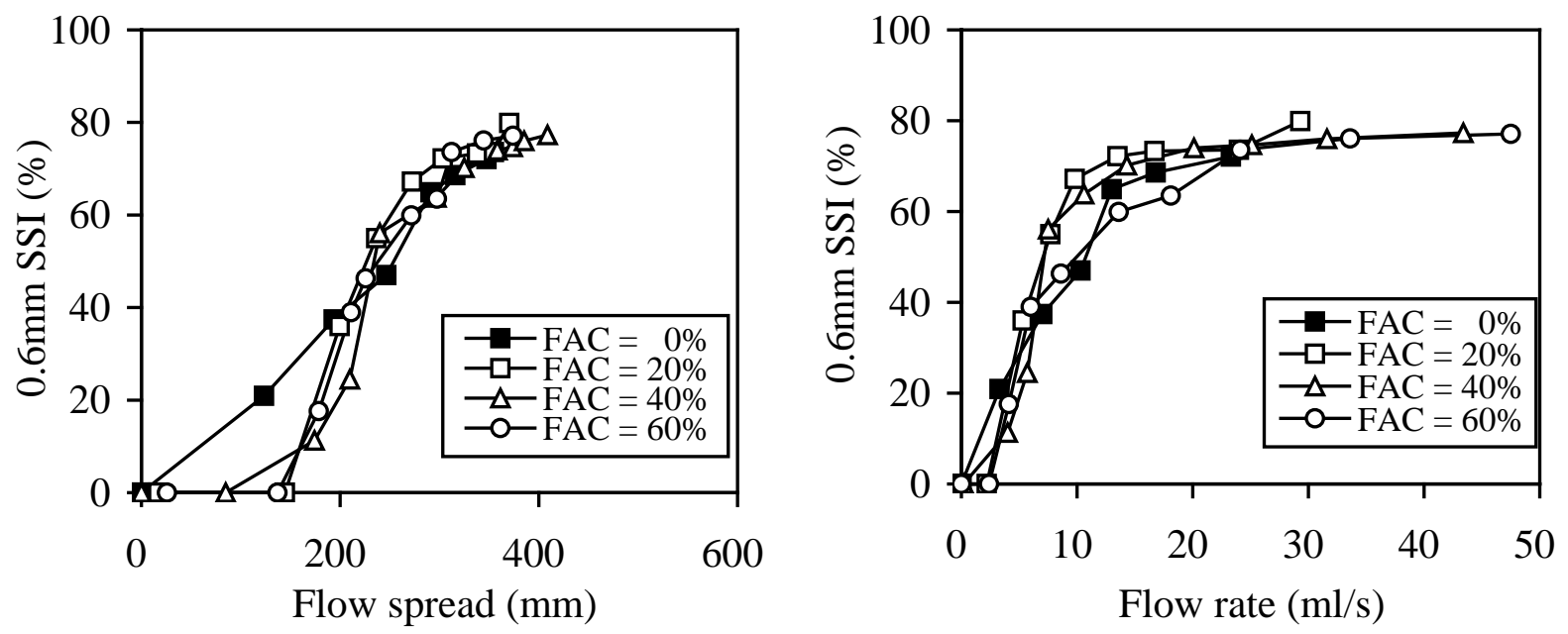

Fig. 6 Effects of FAM on concurrent cohesiveness-flowability performance

\section{Conclusions}

From the experimental findings, the following conclusions can be drawn:

1) The flow spread and flow rate results revealed that at the same W/CM ratio, the flowability always increased as the FAM content increased up to $40 \%$ by volume.

2) The SSI results revealed that at the same W/CM ratio, the cohesiveness always decreased as the FAM content increased up to $40 \%$ by volume.

3) The cohesiveness of cement paste generally decreased with the flowability. The addition of FAM led to a higher cohesiveness at a low flowability while a slightly lower cohesiveness at a high flowability.

\section{Acknowledgement}

This research was financially supported by the PhD Start-up Fund of Natural Science Foundation of Guangdong Province, China (Project No. 2014A030310273) and Science and Technology Project of Foshan City (Project No. 2016AB000031).

\section{References}

[1] H.H.C. Wong, A.K.H. Kwan, Rheology of cement paste: role of excess water to solid surface area ratio, J. Mater. Civ. Eng. 20 (2) (2008) 189-197.

[2] A.K.H. Kwan, H.H.C. Wong, Effects of packing density, excess water and solid surface area on flowability of cement paste, Adv. Cem. Res. 20 (1) (2008) 1-11.

[3] P.-C. Aïtcin, High-Performance Concrete, E \& FN Spon, London and New York, 1998, 591 pp.

[4] M. Wang, D. Jia, P. He, Y. Zhou, Microstructural and mechanical characterization of fly ash cenosphere/metakaolin-based geopolymeric composites, Ceram. Int. 37 (5) (2011) 1661-1666.

[5] Z.D. Li, H.L. Wang, X.N. Wei, X.Y. Liu, Y.F. Yang, W.F. Jiang, Preparation and photocatalytic performance of magnetic Fe3O4@TiO2 core-shell microspheres supported by silica aerogels from industrial fly ash, J. Alloy. Compd. 659 (2016) 240-247.

[6] H. Okamura, M. Ouchi, Self-Compacting Concrete, J. Adv. Concr. Technol. 1 (1) (2003) 5-15. 